\title{
A set approach to the simultaneous localization and map building; application to underwater robots
}

\author{
Luc Jaulin $^{1}$, Frédéric Dabe ${ }^{2}$, Alain Bertholom ${ }^{2}$, and Michel Legris ${ }^{1}$ \\ ${ }^{1}$ E3I2, ENSIETA, Brest \\ ${ }^{2}$ GESMA, Brest \\ No Institute Given
}

\begin{abstract}
This paper proposes a set approach for the simultaneous localization and mapping (SLAM) in a submarine context. It shows that this problem can be cast into a constraint satisfaction problem which can be solve efficiently using interval analysis and propagation algorithms. The efficiency of the resulting propagation method is illustrated on the localization of submarine robot, named Redermor. The experiments have been collected by the GESMA (Groupe d'Etude Sous-Marine de l'Atlantique) in the Douarnenez Bay, in Brittany.
\end{abstract}





\section{Introduction}

This paper deals with the simultaneous localization and map building problem (SLAM) in a submarine context (see [9] for the general SLAM problem). A set membership approach for SLAM (see e.g., [11]) will be considered and it will be shown that this approach leads us to a constraints satisfaction problem (CSP) (see e.g., [8] for notions related CSP and applications) which can be solved efficiently using interval constraints propagation. The efficiency of the approach is illustrated on an experiment where an actual underwater vehicle is involved. In this problem, we try to find an envelope for the trajectory of the robot and to compute sets which contains some detected sea marks (such as mines).

Set-membership methods have often been considered for the localization of robots (see, e.g., [13], [7], in the case where the problem is linear). In situations where strong nonlinearities are involved, interval analysis has been shown to be particularly usefull (see e.g. [12], where one of the first localization of an actual robot has been solved with interval methods). Interval analysis has been shown to be efficient in several SLAM applications (see [4] and [14] where interval analysis has already been used in the context of SLAM for wheeled robots). But the approach is here made more efficient by the addition of constraint propagation techniques. Note that there exist many other robotics applications where interval constraint propagation methods have been successful (see, e.g., [1] for the calibration of robots, [15] for state estimation, [6] for dynamic localization of robots, [10], [17] for control of robots, [3] for topology analysis of configuration spaces, ....).

\section{Robot}

The robot to be considered in our application is an autonomous underwater vehicle (AUV), named Redermor (see Figure 1). This robot, developed by GESMA (Groupe d'Etude Sous-Marine de l'Atlantique), has a length of $6 \mathrm{~m}$, a diameter of $1 \mathrm{~m}$ and a weight of $3800 \mathrm{Kg}$. It has powerful propulsion and control system able to provide hovering capabilities. The main purpose of the Redermor is to evaluate improved navigation by the use of sonar information. It is equipped with a KLEIN 5400 side scan sonar which makes it possible to localize objects such as rocks or mines. It also encloses other sophisticated sensors such as a Lock-Doppler to estimate its speed and a gyrocompass to get its Euler angles.

\section{Method}

In the graphSLAM approach [16], a criterion is built by taking all constraints into account. Then, a local minimization method, such as conjugate gradient, is used to find a good solution of the SLAM problem. Here, we adopt a similar approach, but instead of building a criterion, we cast the SLAM problem into a huge constraints satisfaction 


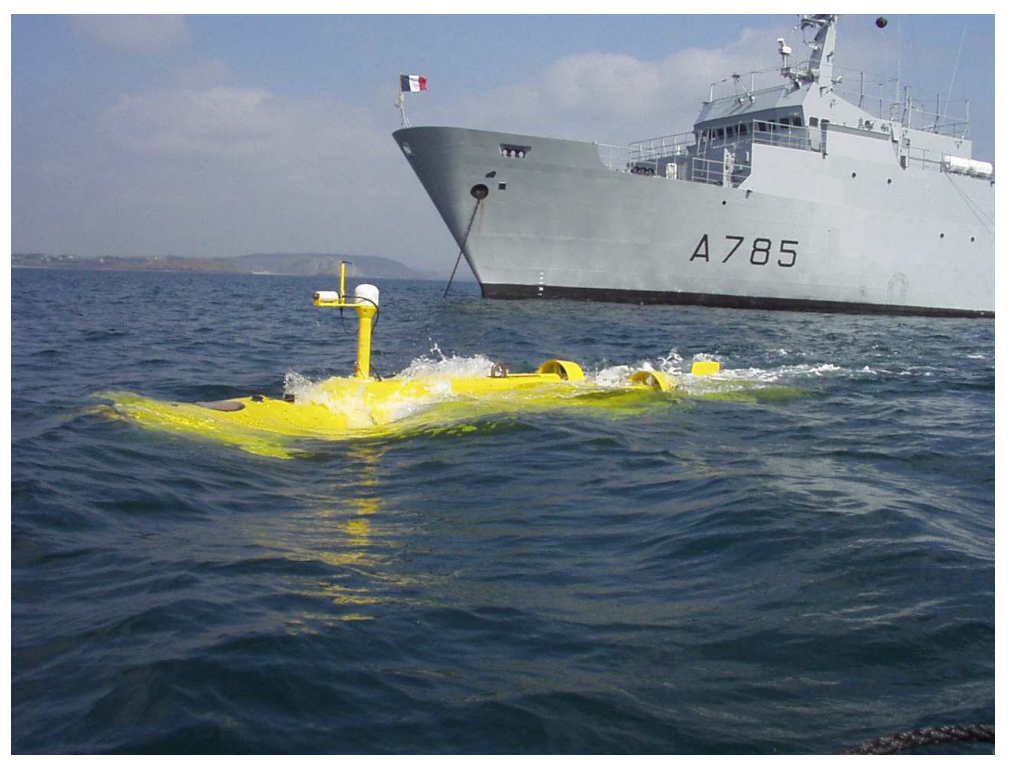

Fig. 1. The Redermor inside the water and the boat from which it has been dropped

problem (CSP). For our problem, these constraints are given below.

$$
\left\{\begin{array}{l}
t \in\{6000.0,6000.1,6000.2, \ldots, 11999.4\}, i \in\{0,1, \ldots, 11\} \\
\left(\begin{array}{l}
p_{x}(t) \\
p_{y}(t)
\end{array}\right)=111120 .\left(\begin{array}{cc}
0 & 1 \\
\cos \left(\ell_{y}(t) * \frac{\pi}{180}\right) & 0
\end{array}\right)\left(\begin{array}{l}
\ell_{x}(t)-\ell_{x}^{0} \\
\ell_{y}(t)-\ell_{y}^{0}
\end{array}\right), \\
\mathbf{R}(t)=\left(\begin{array}{ccc}
\cos \psi_{t}-\sin \psi_{t} & 0 \\
\sin \psi_{t} & \cos \psi_{t} & 0 \\
0 & 0 & 1
\end{array}\right)\left(\begin{array}{ccc}
\cos \theta_{t} & 0 \sin \theta_{t} \\
0 & 1 & 0 \\
-\sin \theta_{t} & 0 \cos \theta_{t}
\end{array}\right)\left(\begin{array}{ccc}
1 & 0 & 0 \\
0 \cos \varphi_{t}-\sin \varphi_{t} \\
0 \sin \varphi_{t} & \cos \varphi_{t}
\end{array}\right) \\
\mathbf{p}(t)=\left(p_{x}(t), p_{y}(t), p_{z}(t)\right), \mathbf{p}(t+0.1)=\mathbf{p}(t)+0.1 * \mathbf{R}(t) \cdot \mathbf{v}_{r}(t), \\
\|\mathbf{m}(\sigma(i))-\mathbf{p}(\tau(i))\|=r(i), \\
\mathbf{R}^{\mathrm{T}}(\tau(i))(\mathbf{m}(\sigma(i))-\mathbf{p}(\tau(i))) \in[0,0] \times[0, \infty] \times[0, \infty], \\
m_{z}(\sigma(i))-p_{z}(\tau(i))-a(\tau(i)) \in[-0.5,0.5]
\end{array}\right.
$$

In these constraints, $\mathbf{p}=\left(p_{x}, p_{y}, p_{z}\right)$ denotes center of the robot, $(\psi, \theta, \varphi)$ denote the Euler angles of the robot, $\sigma(i)$ is the number of the $i$ th detected object, $\tau(i)$ is the time corresponding the $i$ th detection and $\mathbf{m}(j)$ is the location of the $j$ th object. From this CSP, a constraint propagation procedure (see, e.g., [8]) can thus be used to contract all domains for the variables without loosing a single solution.

\section{Results}

A constraints propagation procedure has been used to contract all domains of our CSP. The results obtained are represented on Figure 2. Subfigure (a) represents a punctual estimation of the trajectory of the robot. This estimation has been obtained by integrating the state equations from the initial point (represented on lower part). We have 
also represented the 6 objects that have been dropped at the bottom of the ocean during the experiments. Subfigure (b) represents an envelope of the trajectory obtained using an interval integration, from a small initial box, obtained by the GPS at the beginning of the mission. In Subfigure (c) a final GPS point has also been considered and a forward-backward propagation has been performed up to equilibrium. In Figure (d) the constraints involving the object have been considered for the propagation. The envelope is now thinner and enveloping boxes containing the objects have also been obtained (see Subfigure (e)). We have checked that the unknown actual positions for the objects (that have been measured independently during the experiments) all belong to the associated box, painted black. In Subfigure (f), a zooming perspective of the trajectory and the enveloping boxes for the detected objects have been represented. The computing time to get all these envelopes in less than one minute with a Pentium III. About ten forward-backward interval propagations have been performed to get the steady box of the CSP.

In the case where the position of the marks is approximately known, the SLAM problem translates into a state estimation problem. The envelope for the trajectory becomes very thin and a short computation time is needed. The capabilities of interval propagation methods for state estimation in a bounded error context have already been demonstrated in several applications (see e.g., [5], [2], [1], [6], [8]).

Figure 3 contains the reconstructed waterfall (above) and one zoom (below). Each line corresponds to one of the six seamarks $(i=0, \ldots, 5)$ that have been detected. The gray areas contains the set of all feasible pairs $\left(t,\left\|\mathbf{p}-\mathbf{m}_{i}\right\|\right)$, associated to the migration hyperbola. The twelve small black disks correspond to the detected marks. From each disk, we can get the time $t$ at which the mark has been detected ( $x$-axis), the number of the mark (corresponding to the line), and the distance $r_{i}$ between the robot and the mark ( $y$-axis). Black areas correspond to all feasible $\left(t, r_{i}\right)$. Some of these areas are tiny and are covered by a black disk. Some are larger and do not contain any black disk. In such a case, an existing mark may have been missed by the operator during the scrolling of the waterfall. As a consequence, with the help of Figure 3, the operator could scan again the waterfall and find undetected marks much more efficiently. If the operator (which could be a human or a program) is able to detect at least one more mark, then, the propagation algorithm could be thrown once more to get a thinner envelope for the trajectory, thinner black areas in the reconstructed waterfall and thus a higher probability to detect new marks on the waterfall, ... The operator can thus be seen as a contractor ([8]) inside a constraint propagation process.

\section{Conclusion}

In this paper, we have shown that interval constraints propagation could be applied to solve efficiently SLAM problems. The approach has been demonstrated on an experiment made with an actual underwater robot (the Redermor). The experiment lasted two hours and involved thousands of data. If all assumptions on the bounds of the sensors, on the flat bottom, on the model of the robot, ... are satisfied, then their exists always 


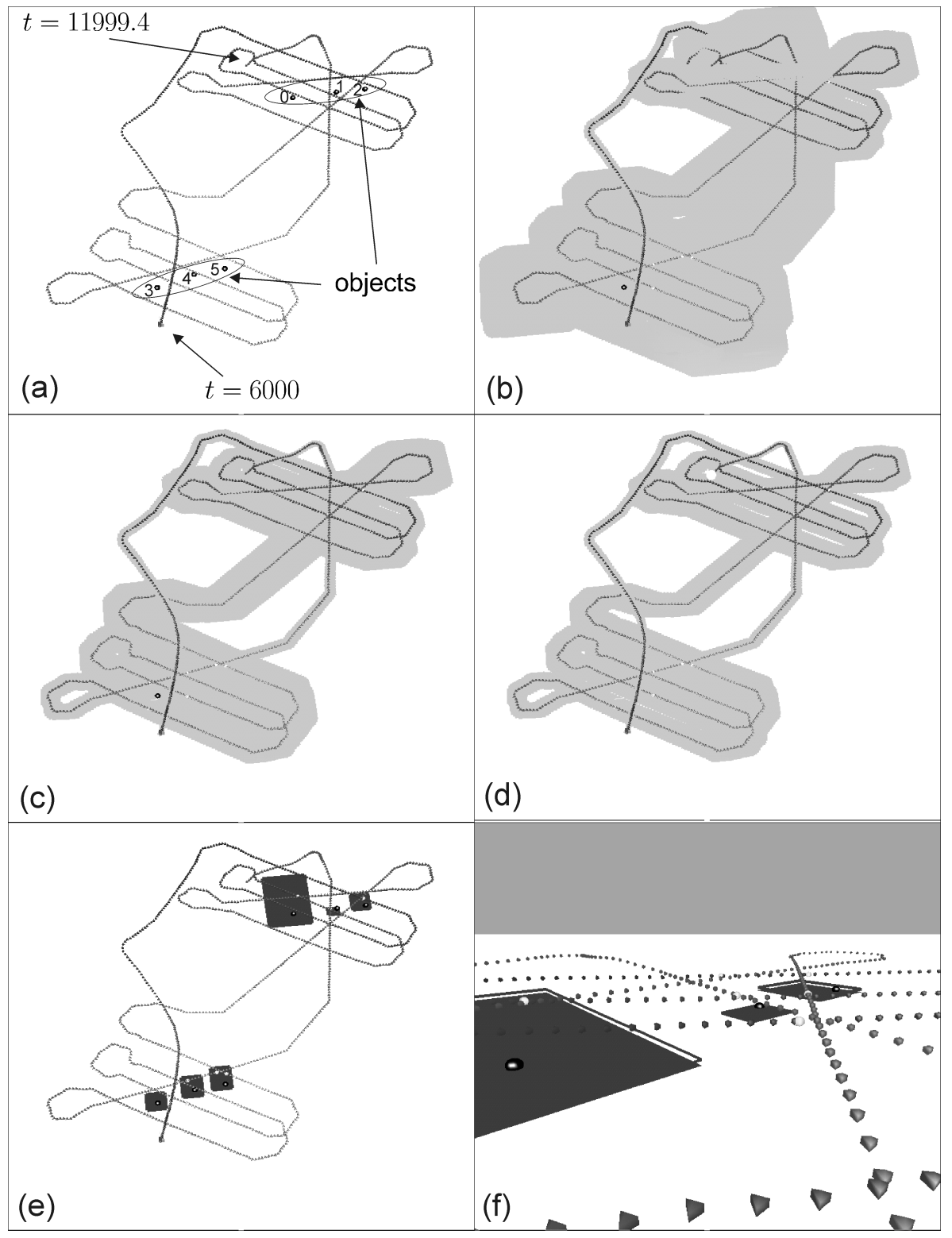

Fig. 2. Results obtained by the interval constraint propagation 


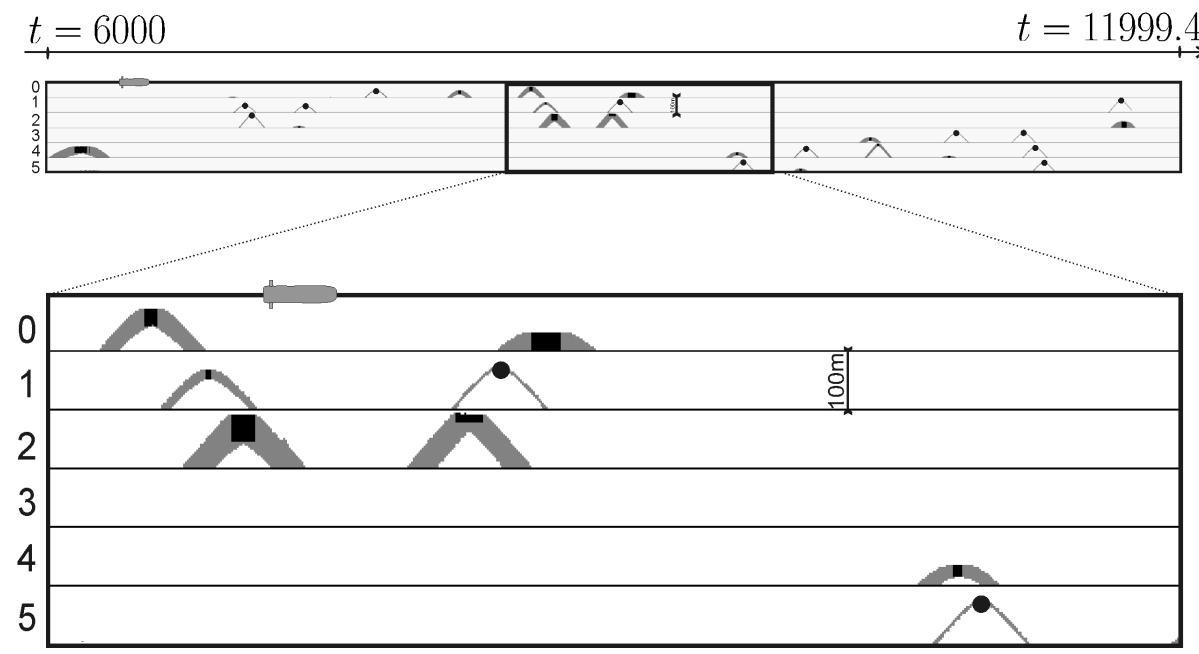

Fig. 3. The reconstructed waterfalls can help to find undetected marks

at least one solution of our problem: that corresponding to the actual trajectory of the robot.

When outliers occur during the experiment, our approach is not reliable anymore and one should take care about any false interpretation of the results. Consider now three different situation that should be known by any user of our approach for SLAM.

Situation 1. The solution set is empty and an empty set is returned by the propagation procedure. Our approach detects that their exists at least one outlier but it is not able to return any estimation of the trajectory and the positions of the marks. It is also not able to detect which sensor is responsible for the failure.

Situation 2. The solution set is empty but nonempty thin intervals for the variables are returned by the propagation. Our approach is not efficient enough to detect that outliers exist and we can wrongly interpret that an accurate and guaranteed estimation of the trajectory of the robot has been done. Other more efficient algorithms could be able to prove that no solution exists which would lead us to the situation 1 .

Situation 3. The solution set is not empty but it does not contain the actual trajectory of the robot. No method could be able to prove that outliers occur. Again, our approach could lead us to the false conclusion that a guaranteed estimation of the trajectory of the robot has been done, whereas, the robot might be somewhere else.

Now, for our experiment made on the Redermor, it is clear that outliers might be present. We have observed that when we corrupt some data voluntarily (to create outliers), the propagation method usually returns rapidly that no solution exists for our set of constraints. For our experiment, with the data collected, we did not obtain an empty set. The only thing that we can conclude is that if no outlier exist (which cannot be guaranteed), then the provided envelope contains the actual trajectory for the robot. 


\section{References}

1. X. BAguenard. "Propagation de contraintes sur les intervalles. Application à l'étalonnage des robots". PhD dissertation, Université d'Angers, Angers, France (2005). Available at: www.istia.univ-angers.fr/ baguenar/.

2. P. Bouron. "Méthodes ensemblistes pour le diagnostic, l'estimation d'état et la fusion de données temporelles." $\mathrm{PhD}$ dissertation, Université de Compiègne, Compiègne, France (Juillet 2002).

3. N. Delanoue, L. Jaulin, And B. Cottenceau. Using interval arithmetic to prove that a set is path-connected. Theoretical Computer Science, Special issue: Real Numbers and Computers 351(1), 119-128 (2006).

4. C. Drocourt, L. Delahoche, B. M. E. Brassart, and A. Clerentin. Incremental construction of the robot's environmental map using interval analysis. Global Optimization and Constraint Satisfaction: Second International Workshop, COCOS 2003 3478, 127-141 (2005).

5. A. GNING. "Localisation garantie d'automobiles. Contribution aux techniques de satisfaction de contraintes sur les intervalles". PhD dissertation, Université de Technologie de Compiègne, Compiègne, France (2006).

6. A. GNING AND P. BonNifait. Constraints propagation techniques on intervals for a guaranteed localization using redundant data. Automatica 42(7), 1167-1175 (2006).

7. E. HALBWACHS AND D. MEIZEL. Bounded-error estimation for mobile vehicule localization. CESA'96 IMACS Multiconference (Symposium on Modelling, Analysis and Simulation) pp. 1005-1010 (1996).

8. L. Jaulin, M. KiefFer, O. Didrit, E. Walter. "Applied Interval Analysis, with Examples in Parameter and State Estimation, Robust Control and Robotics". Springer-Verlag, London (2001).

9. J. J. LeOnard And H. F. DuRRant-Whyte. Dynamic map building for an autonomous mobile robot. International Journal of Robotics Research 11(4) (1992).

10. F. LYDOIRE AND P. POIGNET. Nonlinear predictive control using constraint satisfaction. In "In 2nd International Workshop on Global Constrained Optimization and Constraint Satisfaction (COCOS)", pp. 179-188 (2003).

11. M. D. Marco, A. Garulli, S. Lacroix, and A. Vicino. A set theoretic approach to the simultaneous localization and map building problem. In "In Proceedings of the 39th IEEE Conference on Decision and Control”, vol. 1, pp. 833-838, Sydney, Australia (2000).

12. D. Meizel, O. LÉvêque, L. Jaulin, AND E. WALter. Initial localization by set inversion. IEEE transactions on robotics and Automation 18(6), 966-971 (2002).

13. D. Meizel, A. Preciado-Ruiz, ANd E. HalbwaChs. Estimation of mobile robot localization: geometric approaches. In M. Milanese, J. Norton, H. Piet-LAHANiER, AND E. WALTER, editors, "Bounding Approaches to System Identification”, pp. 463-489. Plenum Press, New York, NY (1996).

14. J. PORTA. Cuikslam: A kinematics-based approach to slam. In "Proceedings of the 2005 IEEE International Conference on Robotics and Automation”, pp. 2436-2442, Barcelona (Spain) (2005).

15. T. RAISSI, N. RAMDANI, AND Y. CANDAU. Set membership state and parameter estimation for systems described by nonlinear differential equations. Automatica 40, 1771-1777 (2004).

16. S. Thrun AND M. MonTEMERLO. The GraphSLAM algorithm with applications to largescale mapping of urban structures. International Journal on Robotics Research 25(5/6), 403-430 (2005).

17. P. H. Vinas, M. A. SAInZ, J. Vehi, AND L. JaUlin. Quantified set inversion algorithm with applications to control. Reliable computing 11(5), 369-382 (2006). 